\title{
An in vitro study of lipid preference in whaleworm (Anisakis simplex, Nematoda, Ascaridoidea, Anisakidae) third-stage larvae
}

\author{
Einar Strømnes
}

Received: 28 November 2013 / Accepted: 26 December 2013 / Published online: 24 January 2014

(C) The Author(s) 2014. This article is published with open access at Springerlink.com

\begin{abstract}
The behavioural response of nematodes to chemical stimuli has been extensively investigated in some freeliving and plant parasitic species. However, in animal parasitic species, little is yet known, particularly in regards to marine forms such as the whaleworm (Anisakis simplex). Previous studies showed that $A$. simplex L3-larvae tend to prefer fish tissue with high lipid content. The intention of this study was to investigate the behaviour of A. simplex L3 in response to different concentrations of fish lipid in further detail. This was done by an in vitro study based on larvae from cod (Gadus morhua). Ten larvae were placed in each of the culture containers containing agar that was separated into three segments of equal size. Three categories of agar were used containing 0,2 and $7 \%$ cod liver oil. A total of 900 larvae were included. The study consisted of three parts: The purpose of experiment I was to establish whether different lipid concentrations influenced the migration pattern at all. Experiment II was intended to examine whether A. simplex L3-larvae were able to actively search for lipids. Experiment III was set up to analyse the shortdistance dispersion of the L3-larvae. Experiment I indicated that the L3-larvae move randomly but do not stop randomly since the tendency to move out of the start area was inversely correlated with lipid concentration. Experiment II indicates that the larvae are almost unable to select areas of high lipid concentrations when more than a few centimetres away. Experiment III showed that the L3-larvae prefer high-fat content and can seek it out over short distances.
\end{abstract}

E. Strømnes $(\bowtie)$

Natural History Museum, University of Oslo, P.O.Box 1172 Blindern, NO-0318 Oslo, Norway

e-mail: einar.stromnes@nhm.uio.no

\section{Introduction}

Even though the presence of Anisakis simplex larvae in fish flesh cause considerable year-on-year economic losses in the fishing industry, little is known about the chemical mechanisms governing the movement of the larvae in the fish hosts. In nematodes, behaviour in relation to chemical stimuli has been extensively studied in some free-living species such as Caenorhabditis elegans and certain species of plant parasites. In these cases, it appears that the amphids are the primary organs involved in responses based on smell or taste (Riga et al. 1995). Far less is known regarding responses to chemical stimuli in animal parasitic nematodes and most studies have been of species in which the larvae actively penetrate the skin. Such larvae are also capable of following gradients, and can register a potential host by means of both chemical and thermal stimuli, as well as mechano-sensorial stimuli (Granzer and Haas 1991; Ashton et al. 1999).

Regarding marine animal parasitic species, even less is known about their response patterns in connection to chemical conditions. However, the general impression in studies dealing with the distribution of $A$. simplex in fish and cephalopods is that they, to a large extent, spread in a random manner throughout these hosts (e.g., Novotny and Uzmann 1960; Wootten and Waddell 1977; Smith 1984; Manfredi et al. 2000).

Young (1972) suggested that A. simplex L3-larvae do not orient themselves according to chemical stimuli, but that the distribution in fish is a result of a random "optimal preencapsulation migratory distance". However, this hypothesis was not supported by Strømnes and Andersen (1998). On the contrary, this study showed that L3-larvae had a certain preference for tissue with high lipid content, whereas the distance from the intestine (the point of entry) was of less importance. In order to further elucidate this issue, the questions of whether the 
observations made in wild fish could be verified under experimental conditions were addressed in the present study.

As opposed to free-living nematodes and the larval stages of terrestrial plant and animal parasitic nematodes, the larvae of A. simplex are spread randomly throughout the marine food web without any influence over which host they enter (e.g., Anderson 2000). Hence, for parasites with this type of life cycle, it is important to be highly flexible in their way of life. A goal-seeking behaviour in terms of localising a new host is, therefore, presumably of little adaptive relevance. Strømnes and Andersen (2003) found that the length of $A$. simplex L3 was significantly correlated with host tissue fat content. Even though it is evolutionarily rational for $A$. simplex $\mathrm{L} 3$ to be a generalist, this may indicate that it is an adaptive advantage to be oriented towards encapsulation in a more fat-rich host tissue.

Anisakis simplex L3 can be found throughout many fish hosts, in all kinds of tissues (Wootten 1978; Smith 1984; Bahool et al. 2012). Nevertheless, Strømnes and Andersen (1998) showed more larvae in lipid-rich fish tissue. Thus, larval distribution may be governed by a behaviour based on random migration coupled with non-random stopping due to some biochemical signal. An evident candidate for a stopping cue is fish lipids. Consequently, it is expected that the L3larvae would show a greater tendency to stop at higher lipid concentrations resulting in a positive correlation between larval abundance and lipid content of the microenvironment. In short, the aim of the present study was to investigate whether A. simplex L3-larvae have the ability to react behaviourally to variations in lipid concentration.

\section{Materials and methods}

Initially, a pilot in vitro study was performed. Here, the capacity for movement in A. simplex L3-larvae turned out to be relatively low: no larva moved more than $10.5 \mathrm{~cm}$ from its starting point. In addition, most of the movement took place within 3-4 days. Based on these results, an experiment with $12 \times 12$-cm culture dishes and a duration of 2 weeks was taken to give a representative picture of the movement pattern of the larvae in question.

The A. simplex L3-larvae used in this study were collected from four specimens of cod (Gadus morhua L.), 80-85-cm long, caught outside Eggum in the Lofoten archipelago in northern Norway $\left(68^{\circ} 22^{\prime} \mathrm{N}, 13^{\circ} 38^{\prime} \mathrm{E}\right)$ in mid-June of 2002 . A sub-sample was collected by first picking medium-sized larvae (about $2.5 \mathrm{~cm}$ ) from the cod livers. These nematodes were put into a $250-\mathrm{ml}$ standard laboratory beaker containing $150 \mathrm{ml}$ of physiological saline solution (distilled water containing $0.9 \% \mathrm{NaCl}$ ). Subsequently, a pair of tweezers was employed to randomly pick up the pre-decided number of specimens. The larvae were kept in a solution of Pepsin A (Fluka, CAS-reg. no. 9001-75-6) and $\mathrm{HCl}$ (Smith and
Wootten 1975), and larvae were left in this solution for $15 \mathrm{~min}$ at $20^{\circ} \mathrm{C}$ to soften the host tissue surrounding them and possibly giving them a stimulus to move. Finally, the remaining host tissue was removed.

An agar solution of $0.7 \%$ (Swelling index 13, Norwegian Medicinal Depot) was found to be optimal regarding the density of the medium. A greater density seemed to inhibit the larvae's moving capability, while a lower content of agar resulted in a culture medium that was too liquid. The agar mixture was based on distilled physiological saline solution.

Three categories of agar were used (Fig. 1): A: agar based on physiological saline solution only (oil-free, i.e. $0 \% \operatorname{cod}$ liver oil); B: agar with $2 \%$ and C: agar with $7 \%$ standardised cod liver oil ("Møllers"TM tran", Norway) added. A maximum value of $7 \%$ was selected since this was found to be the approximate "saturation point" for this type of oil in the agar. However, even at this value, some oil was exuded during the experimental period. An oil concentration of $2 \%$ was selected as a reasonable estimate of a low concentration relative to $7 \%$.

In wild fish, the lipid content of tissues can vary greatly throughout the year depending on temperature and conditions of nourishment. In cod (from where the larvae in this experiment were collected), the liver is the most lipid-rich organ and may contain more than $30 \%$ lipids (Love 1988). This is far above the "saturation point" of the agar used in the present study, and is one reason why it is difficult to relate the current in vitro environment directly to the in vivo environment of the larvae. Hence, the relationship between these two conditions will not be further addressed in this study.

All the culture dishes in the study were filled with a 1.8$2.0-\mathrm{cm}$ layer of agar (see below) and divided into three equalsize segments, each 4-cm wide. Ten L3-larvae were then implanted $1-\mathrm{cm}$ below the surface in the beginning segments. The agar layer consisted of clearly delineated segments, rather than gradients, since this, to a greater degree, mirrors the categorically organised micro-environment in the fish host organs. In an attempt to approximate the thermal conditions in fish from the sampling area at the current time of the year (Institute of Marine Research, Norway, http://www.imr.no/ forskning/forskningsdata/stasjoner/), the culture dishes were placed in a room with a constant temperature of $12{ }^{\circ} \mathrm{C}$ and darkness. At the end of the experimental period, the culture dishes were placed on a light table, and the distribution pattern of the nematodes was registered.

The investigation was divided into three parts (Fig. 1). In order to investigate whether lipid concentration differences influence the movement pattern of L3-larvae, one experiment (experiment I) was designed with three groups of culture dishes, each containing only one of the three categories of agar (A, B or C). If A. simplex L3-larvae do not respond to cod liver oil as a chemical cue, one would expect a random distribution in all three culture groups. In the following, the group of culture dishes containing only oil-free agar, category 
Fig. 1 a, b, c The experimental designs: culture dishes with three segments of agar and the position of the Anisakis simplex L3-larvae (short irregular lines) at the start of the experiments. The L3-larvae were implanted $1-\mathrm{cm}$ below the surface and maintained in darkness at $12^{\circ} \mathrm{C}$ for 14 days. Three categories of agar were used: $A=0 \%$ oil; $B=2 \%$ oil and $C=7 \%$ oil. Experiment I: The dishes were divided into three groups (groups 1, 2 and 3), each with one agar category only, either $A, B$ or $C$. The L3-larvae were implanted along the centre line of the middle segment. Experiment II: The dishes were divided into two groups (groups 4 and 5). The L3-larvae were in the oil-free A segment at one end of the dish, while the positions of segments $\mathrm{B}$ and $\mathrm{C}$ were varied. $\mathrm{c}$ Experiment III: Consisted of one group (group 6). The larvae were, as in experiment I, implanted along the centre line of the middle segment a Experiment I

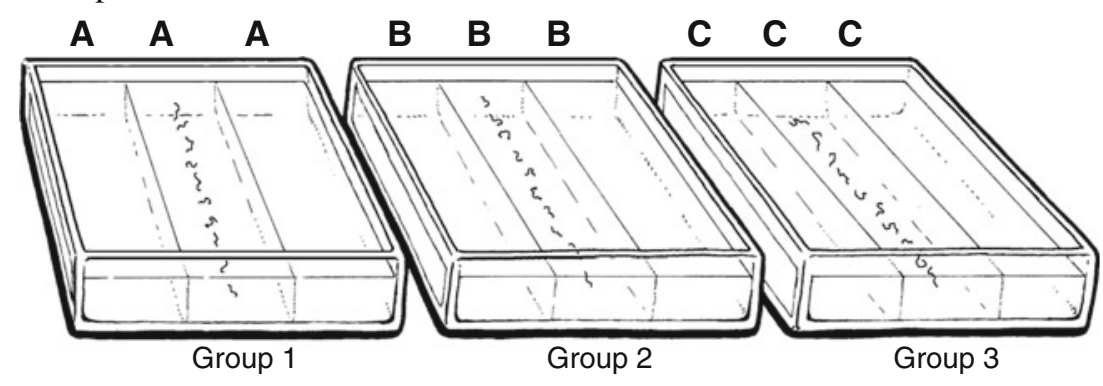

b Experiment II

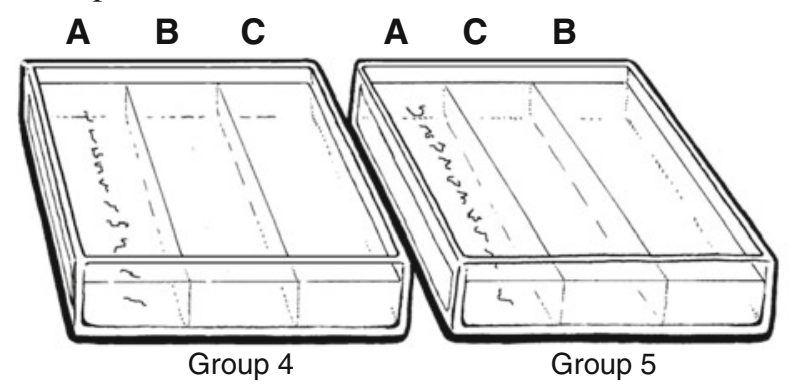

C Experiment III

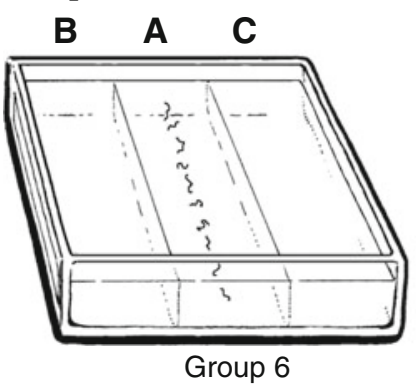

A, is designated as group 1, the group with only agar of category B as group 2, and the group with only agar of category $\mathrm{C}$ as group 3.

Although the agar for experiment I was made in one batch, it was cut into three equally wide segments after hardening in the dishes in order to create conditions as similar as possible to the conditions in experiments II and III, where each culture dish contained all three categories (A, B and C) of agar (see below and Figs. 1 and 2). In experiment I, the start segment was always the middle segment. Here, and throughout the study, the larvae were placed along the centre line of this segment. Experiment I was repeated with ten parallels and a total of 300 larvae.

In order to investigate whether A. simplex L3-larvae are capable of seeking out the most lipid-rich microhabitats available, experiment II contained two different culture groups (Fig. 1b). In both groups, the starting point for the larvae was the oil-free A segment, localised at one end of the culture dishes. Then, the relative positions of the oil-containing segments were switched, so that in one group, the low- concentration segment B bordered segment A. On the other hand, in group 5, the high-concentration segment $\mathrm{C}$ bordered A. This gave the segment sequence A B C and A C B for groups 4 and 5 , respectively. In experiment II, the L3-larvae were placed along the centre line of the A segment.

If L3-larvae are capable of seeking out the most lipid-rich microhabitat within their radius of action, in experiment II, one should expect that a higher proportion of larvae would be localised in the $\mathrm{C}$ segment compared to the $\mathrm{B}$ segment regardless of the different distances to the $\mathrm{C}$ segment, groups 4 and 5 , respectively. In contrast, if the larvae do not have the capacity to respond to different oil concentrations, one would expect a distribution corresponding to that of experiment I, group 1 .

To achieve additional information about the lipid-seeking behaviour of the L3-larvae, a third experiment (experiment III) was designed: The starting segment A was placed in the centre of the culture dishes, with segments $\mathrm{B}$ and $\mathrm{C}$ on either side. Thus, the distance factor that had been introduced in experiment II was eliminated. This group of culture dishes (segment order B A C), will subsequently be designated as 
Fig. 2 Mean numbers of Anisakis simplex L3 larvae in the agar segments from the six culture groups by the end of the study.

Experiment I (light grey columns), where the L3 larvae were implanted along the centre line of the middle segment in all the dishes. Experiment II (medium grey) and experiment III (dark grey), where the L3 larvae were placed along the centre line of the oil-free A segment. $A=0 \%$ oil, $B=2 \%$ oil and $C=7 \%$ oil. Error bars represent \pm standard error (SE)

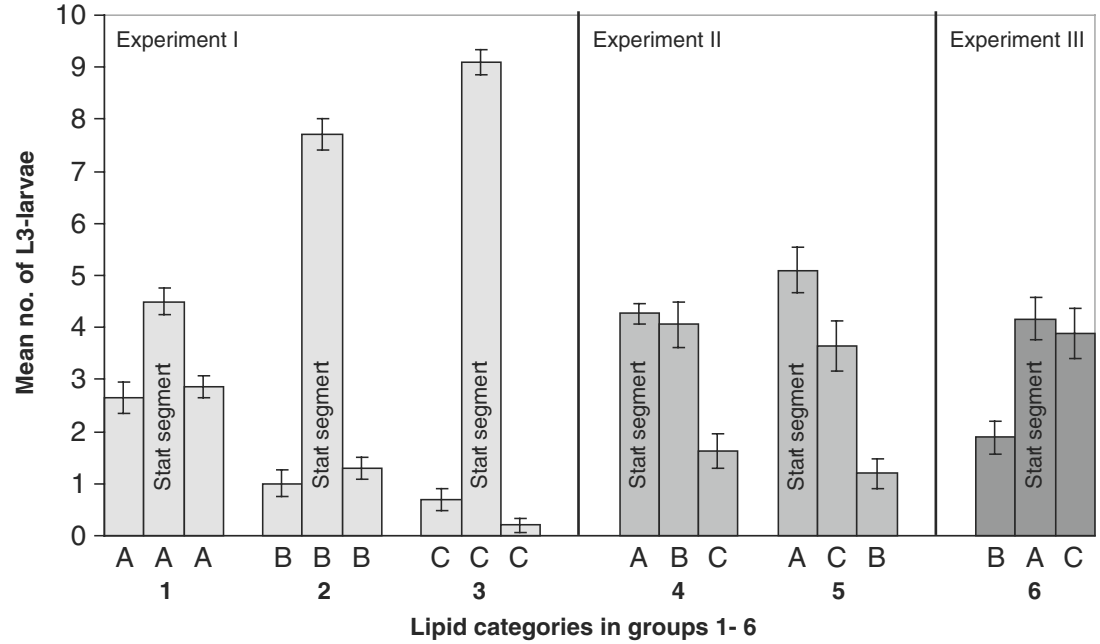

group 6. Here, the L3-larvae were again placed along the centre line of the oil-free segment A. If L3-larvae prefer a high concentration of lipids, one would expect that more larvae in group 6 migrated to segment $\mathrm{C}$ than to segment $\mathrm{B}$.

Experiments II and III were repeated with 20 parallels since the variance in these experimental designs were greater than in experiment I. Thus, experiment II contained 400 larvae, while experiment III (i.e. group 6) contained $200 \mathrm{~A}$. simplex L3-larvae.

To prepare the cultures containing agar with different oil concentrations (experiments II and III), the dishes were first filled with the oil-free agar (category A). The A section was then cut away to make room for a segment of category B agar. Once this mixture had hardened, the last section was cut away and filled with category $\mathrm{C}$ agar. In an attempt to reduce seeping of exuded oil to bordering segments, the oil-free A segments were made 2-cm thick, while the $\mathrm{B}$ and $\mathrm{C}$ segments were 1.9- and $1.8-\mathrm{cm}$ thick, respectively.

To determine whether the number of L3-larvae was significantly different in the various segments, both within a group and between the groups $1-6$, the data were analysed using the general linear models with a binomial distribution (S-plus). In those cases where the larvae started from the middle segment, the non-parametric Wilcoxon paired sample test (ANOVA) was used since this test is more robust than a paired $t$ test.

\section{Results}

The distribution of L3-larvae at the end of the experiment is summarised in Fig. 2. Only one dead larva (in experiment II) was registered at the end of the study.

For group 1 (i.e. $0 \%$ oil in the entire culture), an average of 4.5 out of 10 larvae were localised in the starting segment at the end of the experiment. The two bordering segments in group 1 both contained an average of three larvae. The culture dishes in group 2 (i.e. $2 \%$ oil in the entire culture) clearly had more larvae in the starting segment (7.7), and an average of about 1 larva in the bordering segments. In group 3 (i.e. 7 \% oil in the entire culture), very few larvae in this most lipid-rich environment had moved away_ or had relocated and moved back-from their starting segment. Concerning group 3, an average of 9.1 specimens was localised in the starting segment in the middle of the culture dish by the end of the experiment. The number of L3 found in the starting segments of experiment I was significantly different (deviance $=65.53, D F=2$, $p<0.001$ ). Even the results from groups 2 and 3 , showing the least prominent distinction, were significantly different (deviance $=7.51, D F=1, p=0.006$ ).

In experiment II, the start segment was positioned at one end of the culture dishes. Group 4, with the segment order A B $\mathrm{C}$, showed a decreasing number of larvae with increasing distance from start segment $\mathrm{A}$. The average number of larvae remaining in segment $\mathrm{A}$ was 4.3. The average number of larvae in the bordering segment B was $4.05 \mathrm{~L} 3$, and in the C segment, at the most distant end of the dish, it was 1.63 L3.

The distribution in group 5 (A C B) showed a similar pattern as that of group 4, with a decreasing number of L3 from the starting segment towards the far end of the dish. Group 5 had more larvae in the starting segment (5.1), but somewhat fewer in the bordering $\mathrm{C}$ segment (3.65) and the most distant B segment (1.2).

These differences in groups 4 and 5 were not significant (ANOVA, $p>0.20$ ). There was, however, a tendency to a greater concentration of larvae in segment A, group 5 than in group 4 (deviance $=2.79, D F=1, p=0.094$ ). The number of larvae in segment B, group 4 was significantly higher than in the left and right segments in group $1(p=0.003)$. The number of larvae in the $\mathrm{C}$ segment, group 4 was significantly less than in the left and right segments in group 1 . These differences were also found to be significant with $p=0.005$ and $p=0.002$, respectively.

In experiment III (group 6, B A C), where the distance from the starting segment to each of the oil-containing segments (2 
and $7 \%$ ) was equal, a significantly higher number of L3larvae had migrated to the $\mathrm{C}$ segment than to the $\mathrm{B}$ segment ( $p=0.007$, one-tailed Wilcoxon signed-rank test).

\section{Discussion}

Among the few papers addressing the question of mechanisms behind the distribution pattern in marine parasitic nematodes L3-larvae in fish, Young (1972) proposed a hypothesis of an "optimal pre-encapsulation migratory distance". The suggestion here is that the infestation of anisakid larvae in fish muscle (where they cause a medical problem) would decrease relative to the infestation in the viscera as the size of the fish increases, since the distance from the digestive tract to the muscle tissue would consequently increase. However, Strømnes and Andersen (1998) found that the distribution between tissues was relatively independent of the fish size, and that the distribution pattern also varied considerably among the three species studied (cod, saithe and redfish). On the other hand, these variations seemed connected with the distribution of lipids in the various host species, indicating that this matter required a more elaborate explanation than that of a random and distance-based migration within the host. A plausible explanation might be that the distribution is influenced by the chemical conditions of the microhabitat where ample access to lipids is preferred.

One factor supporting this explanation is the correspondence found by Strømnes and Andersen (2003) between the lipid content of three tissue categories, muscle, liver, other viscera and the size of the $A$. simplex larvae. The L3-larvae found in tissue with high levels of lipids seemed to exhibit a faster growth rate than larvae found at lower lipid levels. If the size of the larvae is presumed advantageous for survival, this may also indicate that a certain capability to actively seeking nutrient-rich microhabitats is beneficial to the species in an evolutionary sense.

The results from experiment I indicate that larval mobility diminished as the oil concentration increased. In a situation where the microhabitat contains little or no lipids, as in group 1, the L3-larvae are apparently stimulated to increase their mobility and actively seek new and possibly better microhabitats. As access to lipids increases, as in groups 2 and 3, the need to seek microhabitats with higher levels of lipids seems to be diminished, and fewer larvae are triggered to move away from their start segment.

In all six combinations of segments, a considerable percentage of the larvae did not move away from their starting segment. The size of the seemingly immobile fraction remaining in the starting segment was constant (45-50\%) in all the groups containing oil-free segments (groups 1 and 4-6). The larvae were not monitored systematically during the study period, so at least some of the specimens may have been visiting the neighbouring segments and then had returned to the starting segment.

By the end of the experiment, there was an approximately equal number of larvae in the starting segment and in the bordering B segment in group 4 (segment order A B C), while the number of larvae in the $\mathrm{C}$ segment was significantly lower. If $A$. simplex L3-larvae are not capable of goal-seeking behaviour, the distance involved, with the starting segment and the C segment at opposite ends of the dish, could offer an explanation for this result.

In group 5 (A C B), the L3-larvae demonstrated a distribution pattern largely congruent with the pattern in group 4. However, there was a tendency in the material to find more L3-larvae in the A segment in group 5 than in group 4. Assuming that the oil to some extent diffuses from one segment to another, this observed difference between groups 4 and 5 may be a reasonable result. The larvae in the start segment in group 5 would have access to more oil than those in group 4, since the A segment in group 5 borders on the oilrich $\mathrm{C}$ segment, while the A segment in group 4 borders on the less oil-rich B segment.

In group 6 (B A C), where the start segment was localised in the middle of the culture dishes, distance was largely eliminated as a variable. Here, significantly more larvae had moved to the $\mathrm{C}$ segment and then to the B segment. This tendency of more larvae preferring the microhabitat with the highest lipid content is expected if $A$. simplex-L3 have a preference for oil, and do not only move randomly from their start point.

The results from experiment I, thus, demonstrate that an increasing concentration of fish oil will reduce the $A$. simplex L3-larvae's tendency to move. In other words, an oil deficit will stimulate migrating behaviour, while this inclination gets weaker at higher oil concentrations as the stop signal gets stronger. Experiment II further indicates that the larvae were not able to examine the entire area available and choose the most favourable segment within the period of time covered by this study.

Nevertheless, experiment III shows that if the larvae, when placed in an environment with little or no fat, are given a choice between areas with different concentrations of fat, they do tend to prefer the most oil-rich environment. These factors indicate that $A$. simplex L3-larvae move randomly, but do not stop randomly, since their tendency to migrate is increasingly inhibited, or their inclination to stop is incited, as the concentration of oil increases.

In experiment I, the distribution pattern observed in group 1 exhibited a random distribution of L3, which could be explained if the migrating larvae moved with random speed and direction, without being stimulated to stop. Similarly, it may be suggested that the distribution in groups 2 and 3 can be explained by an increasing number of larvae experiencing a sufficient inhibition of their inclination to move with an increasing concentration of lipids. Regardless of the actual mechanism, the net migration distance will decrease. 
In experiment II, group 4, the percentage of L3 in the starting segment was approximately equal to the A segment in experiment I, group 1, and the reason for this appears to be the same. In the bordering $\mathrm{B}$ segment, there were an approximately equal number of larvae as found in the A segment. This number was also significantly higher than in the segments bordering the A segment in group 1 , while the number in the $\mathrm{C}$ segment was significantly lower than both. According to the present hypothesis this is a reasonable result. As migrating L3-larvae arrive in segment $\mathrm{B}$, the oil concentration increases, and the probability of the larvae stopping, increases. In this way, the larvae would accumulate in segment B. This also implies that an oil concentration of $2 \%$ is sufficient to make the presumed inhibiting effect clearly noticeable. However, although the inclination may be decreased, some larvae may manage to reach the more distant $\mathrm{C}$ segment, where the probability of migration inhibition is even greater. One indication of this may be that in group 4, where the most distant segment contains the most oil (C); there are more larvae than in the most distant segment in group 5, even if this difference is not significant. In Group 5, the mechanisms will probably be of a similar nature. In this group, slightly fewer larvae move to the most distant segment, and additionally, fewer move out of their starting segment.

In the single group of experiment III (group 0), significantly more larvae were found in the most oil-rich segment $C$ than in the less oil-rich segment B. The observed distribution lends support to the process described by the present hypothesis. Those larvae that through random movement arrive in segment $\mathrm{B}$, will probably have their inclination to move inhibited, and thus they will stop or reduce their velocity. Larvae that do not stop completely, might to some extent, move back to the starting segment and from there over to segment $\mathrm{C}$. There, the oil concentration is so high that, as shown in group 3, the probability of further movement becomes very low. For those larvae that by chance arrived first in segment $\mathrm{C}$, there is a correspondingly small probability of further movement. In this way, more larvae might accumulate in segment $\mathrm{C}$ than in segment $\mathrm{B}$.

The present study supports that A. simplex L3-larvae, in accordance with Young (1972), radiate randomly out from the digestive tract of the fish host. However, contrary to Young's optimal pre-encapsulation migratory distance hypothesis, the A. simplex L3-larvae seem to actively relate to their chemical environment by, at the very least, sensing the fat concentration in their habitat. This suggests a tendency towards reduced migration at higher levels of oil concentration implying an increased probability of L3-larvae accumulating in high-fat tissues of the host. This non-random distribution of $A$. simplex L3-larvae corresponds to findings in wild fish outlined in the introduction (Strømnes and Andersen 1998). This is also in accordance with Sukhdeo and Sukhdeo $(1994,1997)$ who found that endoparasites, living in a very predictable environment like the inside of a fish host, orient themselves by use of chemical cues in a fixed action pattern and not by following gradients.
Acknowledgments Special thanks to Ane Vollsnes, University of Oslo, for useful discussions and comments on the manuscript throughout the writing process. Thanks also to Professor Odd Halvorsen and Professor Phlip Harris, Natural History Museum, UiO, for contributions to early drafts of the paper. Finally, thanks to Petter Bøckman, NHM, UiO, for drawing the culture dishes in Fig. 1.

Open Access This article is distributed under the terms of the Creative Commons Attribution License which permits any use, distribution, and reproduction in any medium, provided the original author(s) and the source are credited.

\section{References}

Anderson RC (2000) Nematode parasites of vertebrates, their development and transmission, 2nd edn. CABI, Wallingford

Ashton FT, Li J, Schad GA (1999) Chemo- and thermosensory neurons: structure and function in animal parasitic nematodes. Vet Parasitol 84:297-316

Bahool QZM, Skovgaard A, Kania P, Haarder S, Buchmann K (2012) Microhabitat preference of Anisakis simplex in three salmoid species: immunological implications. Vet Parasitol 190:489-495

Granzer M, Haas W (1991) Host-finding and host recognition of infective Ancylostoma caninum larvae. Int J Parasitol 21(4):429-440

Love RM (1988) The food fishes: their intrinsic variation and practical implications. Farrand Press, London

Manfredi MT, Crosa G, Galli P, Ganduglia S (2000) Distribution of Anisakis simplex in fish caught in the Ligurian Sea. Parasitol Res 86:551-553

Novotny AJ, Uzmann JR (1960) A statistical analysis of the distribution of a larval nematode (Anisakis sp.) in musculature of chum salmon (Oncorhynchus keta-Walbaum). Exp Parasitol 10:245-262

Riga E, Perry RN, Barret J, Johnston MRL (1995) Investigation of the chemosensory function of amphids of Syngamus trachea using electrophysiological techniques. Parasitol 111:347-351

Smith JW (1984) The abundance of Anisakis simplex L3 in the bodycavity and flesh of marine teleosts. Int J Parasitol 14:491-495

Smith JW, Wootten R (1975) Experimental studies on the migration of Anisakis sp. larvae (Nematoda: Ascaridida) into the flesh of herring, Clupea harengus L. Int J Parasitol 5:133-136

Strømnes E, Andersen A (1998) Distribution of whaleworm (Anisakis simplex, Nematoda, Ascaridoidea) L3 larvae in three species of marine fish; saithe (Pollachius virens (L.)), cod (Gadus morhua L.) and redfish (Sebastes marinus (L.)) from Norwegian waters. Parasitol Res 84:281-285

Strømnes E, Andersen K (2003) Growth of whaleworm (Anisakis simplex, Nematodes, Ascaridoidea, Anisakidae) third-stage larvae in paratenic fish hosts. Parasitol Res 89:335-341

Sukhdeo MVK, Sukhdeo SC (1994) Optimal habitat selection by helminths within the host environment. Parasitology 109(Suppl.):S41-S55

Sukhdeo SC, Sukhdeo MVK, Black MB, Vrijenhoek RC (1997) The evolution of tissue migration in parasitic nematodes (Nematoda, Strongylida) inferred from a protein-coding mitochondrial gene. Biol J Linn Soc 61:281-298

Wootten R (1978) The occurrence of larval anisakid nematodes in small gadoids from Scottish waters. J Mar Biol Assoc UK 58:347-356

Wootten R, Waddell IF (1977) Studies on the biology of larval nematodes from the musculature of cod and whiting in Scottish waters. J Cons Int Explor Mer 37:266-273

Young PC (1972) The relationship between the presence of larval anisakine nematodes in cod and marine mammals in British home waters. J Appl Ecol 9:459-485 